\title{
Los modos de las políticas de la Integración Centroamericana
}

\author{
Francisco Santos Carrillo \\ Universidad Loyola Andalucía \\ Investigador de la Fundación ETEA para el Desarrollo y la Cooperación \\ francis.santos@fundacionetea.org
}

Fecha de recibido: 30 de junio de 2016 / Fecha de aprobación: 25 de julio de 2016

\section{Resumen}

Este trabajo revisa los modos en que se desarrollan las acciones de política regional en el Sistema de Integración Centroamericano (SICA). El impulso de esta agenda de políticas de integración positiva materializa un incipiente proceso de gobernanza regional que tiene como protagonista a las instituciones del SICA, con el apoyo de las coaliciones de grupos de interés conformados y de sus socios de la cooperación internacional, entre los cuales juega un papel destacado la Unión Europea (UE). El proceso de implementación evidencia la existencia de importantes obstáculos que superar, pero demuestra su factibilidad política, jurídica e institucional. Lo más importante es que suponen una oportunidad para afrontar retos comunes en materia de desarrollo aprovechando el creciente grado de interdependencia. Al mismo tiempo, otorga a la integración un papel de mayor relevancia como instrumento de desarrollo.

\section{Palabras Clave}

Políticas regionales / integración regional / regionalismo / gobernanza regional

\section{Abstract}

This paper reviews the ways in which the actions of regional policy in the Central American Integration System (SICA) develop. The impetus for the policy agenda for positive integration materializes an incipient process of regional governance that features the institutions of SICA, supported by coalitions of groups formed interest and partners of international cooperation, including plays an important European Union (EU) role. The implementation process reveals the existence of important obstacles to overcome, but it demonstrates its political, legal and institutional feasibility. The most important thing is to provide an opportunity to address common challenges in development taking advantage of the increasing degree of interdependence. At the same time, the integration gives a greater role as a development tool. 
Regional public policies / regional integration / regionalism / regional governance

\section{Tabla de contenido}

Introducción 2. El debate sobre las Políticas Regionales de la Integración 3. El marco de las Políticas regionales del SICA 4. Los modos de las Políticas del SICA 4.I. Los modos de las políticas de la Unión Europea (UE), 4.2. Una sistematización de las políticas del SICA 5. Conclusiones 6.Biliografía

\section{Introducción}

La integración regional es una dimensión muy presente en el debate del desarrollo en América Latina. No obstante, pese al permanente discurso integracionista, ninguno de los proyectos de la integración latinoamericana ha conseguido definir una verdadera agenda de integración positiva que permitiera incrementar sustancialmente los niveles de interdependencia entre países miembros (Tinbergen, 1957; Scharpf, 2000). Se crearon estructuras políticas e instituciones regionales, pero no se dotaron de políticas.

Como algunos autores han puesto de relieve, no está claro si las acciones de política en el marco de la integración contribuyen al desarrollo en sus distintas dimensiones, o si el nivel regional es apropiado para la provisión de bienes públicos (Best, 2006). La integración actual es un fenómeno complejo a causa de su naturaleza heterogénea, multidimensional y multinivel, incluso en los diseños institucionales abiertamente intergubernamentales (De Lombaerde et al., 2006). No es de extrañar, por tanto, que no haya sido posible alcanzar los consensos necesarios acerca de los beneficios de la integración, especialmente si los acuerdos de integración eran conformados por países en vías de desarrollo, en lo que ha venido a denominarse esquema sur-sur (Schift y Winter, 2004). En el contexto internacional, finalmente, aunque los patrones de la geoeconomía latinoamericana registraron cambios sustanciales, los principales socios de la región han permanecido en los márgenes del paradigma neoliberal, que se ha mostrado irreductible a lo largo de estos años con respecto a su defensa del libre comercio. Junto a otros factores de carácter histórico, político y cultural menos evidentes aunque sobradamente conocidos, los argumentos anteriores contribuyen a entender las reticencias de los gobiernos a la hora de mostrar su voluntad política en favor de la integración.

En Centroamérica, el proyecto integracionista registra algunos logros notables (Caldentey del Pozo, 20I4; Santos y Pozo, 2013; Pellandra y Fuentes, 20II), que superan el desempeño de los proyectos sudamericanos quedando, no obstante, oscurecidos por la menor relevancia económica de sus estados miembros. Registra el mayor incremento relativo de los intercambios intrarregionales, propiciando avances en la regionalización de la actividad productiva y una mayor interdependencia entre sus capitales (Segovia, 2005; Bull, 2012). Y ha sido un instrumento activo en la búsqueda de una mayor inserción internacional de la región. Pero no fue capaz, sin embargo, de crear el marco económico necesario para la generación de mayores tasas de crecimiento en los países miembros (Rueda Junquera y Sánchez Ancochea, 2009; Rueda Junquera, 2014). Tampoco pudo erigirse como eje articulador de los cambios sustanciales que se produjeron a nivel político, social, económico y demográfico (Sánchez-Ancochea y Martí-Puig, 20l4), de tal manera que pudiera contribuir a paliar los bajos niveles de desarrollo que vienen caracterizando la historia centroamericana (Bulmer-Thomas, 1987; Lehoucq, 2012). Antes al contrario, la debilidad institucional que le ha caracterizado durante todos estos años consiguió proyectar una imagen de permanente cuestionamiento.

Hay dos puntos de inflexión en la historia reciente de la integración centroamericana. En primer lugar, el impulso que recibe la integración a partir del huracán Mitch como evidencia de la necesidad de enfrentar conjuntamente los retos de la región. En segundo lugar, la celebración por parte de los países centroamericanos y la cooperación 
internacional de los Grupo Consultivo para la Reconstrucción y la Transformación de América Central en Washington y Estocolmo y el el Grupo Consultivo de Madrid para la transformación y modernización de Centroamérica que se celebró en marzo de 200I. Estos dos hechos marcan una etapa de recuperación del proceso a partir del año 2002 que se apoya en la legitimación de la acción regional que supone la estrategia regional de transformación y modernización basada en el principio de responder conjuntamente a desafíos determinantes para la reducción de las vulnerabilidades de los países centroamericanos, pero inabordables desde enfoques nacionales. El enfoque regional otorgaba un notable protagonismo a la integración regional.

La integración aprovechó ese impulso y los años 2002 a 201 I estuvieron marcados por las reformas institucionales, por el diseño de un marco de agendas regionales y por el apoyo intenso y creciente de la cooperación internacional que encontró en el SICA un ámbito de cooperación internacional complementario al nacional y local.

La crisis de Honduras de junio de 2009 frenó este desarrollo y se combinó con los impactos de una crisis múltiple al sumarse las tensiones fronterizas, la crisis económica internacional y sus efectos en las fuentes de financiación de la región (remesas, exportaciones y maquila), y la crisis ambiental y el recurrente impacto de algunos desastres naturales. A ello se sumó también la crisis de seguridad que progresiva e implacablemente ha convertido a Centroamérica en una de las regiones más violentas del mundo. El sufrimiento y la descomposición institucional y social que la violencia y el crimen organizado provocan en Centroamérica (especialmente en los 3 países del triángulo norte) es fácilmente perceptible siguiendo la coyuntura de la región a través de sus medios de comunicación o revisando la bibliografía sobre el tema o los informes regionales que recientemente han publicado los organismos internacionales sobre este tema (Banco Mundial 201 I y PNUD 2009).

Dos procesos recientes han renovado el rol emergente de la integración en Centroamérica. Uno es la Conferencia Internacional de apoyo a la Estrategia de Seguridad de junio de $201 \mathrm{l}$ de gran impacto en la Comunidad internacional. El segundo proceso es el relanzamiento de la integración centroamericana, promovido por los gobiernos de El Salvador y Guatemala a partir de la Cumbre extraordinaria celebrada el 20 de julio de 2010 en El Salvador.

En un intento de impulsar el proceso, en diciembre de 2010 los presidentes centroamericanos aprobaron la agenda del "Relanzamiento de la Integración Centroamericana". Sustentada en torno a cinco ejes sectoriales (seguridad democrática, prevención y mitigación de desastres naturales y efectos del cambio climático, integración social, integración económica y fortalecimiento institucional), esta agenda ha conseguido articular un catálogo de acciones de política regional que supera los límites de la integración económica y retoma el compromiso inicial con el desarrollo (Caldentey, 2014; Santos y Pozo, 20I3). La región ya contaba con un conjunto de agendas y políticas que los países e instituciones de la región venían promoviendo y aprobando desde el año 2002 tras el Mitch y el Grupo Consultivo de Madrid .La relevancia de este relanzamiento nace de que propone organizar una agenda contenida y factible sobre 5 prioridades y explora instrumentos metodológicos para facilitar la concreción de los acuerdos, la definición de las competencias derivadas de él y el seguimiento de su ejecución.

El impulso en materia de políticas regionales ofrece una oportunidad para combatir algunos de los obstáculos al desarrollo de la región desde las instituciones de la integración. Este impulso podría aspirar a ajustar y contrarrestar el impacto del nuevo mercado regional y sus dinámicas, muy distintas a las de los mercados nacionales anteriores; y podría articular procesos de gobernanza alternativos de tipo intergubernamental, interinstitucional y multinivel, de tal forma que rompieran el monopolio de los gobiernos nacionales en relación con los actores y en con la formulación de políticas, dando lugar a nuevas coaliciones entre las instancias instituciones regionales y los actores locales y sectoriales. Este tipo de políticas contribuye también a alejar la idea de una integración orientada a los intereses económicos de los sectores empresariales.

No obstante, el marco regional donde deben desarrollarse estas políticas se enfrenta a contradicciones importantes entre el marco jurídico e institucional, un intergubernamentalismo que dificulta los consensos y una 
estructura política regional cuya sostenibilidad y agenda dependen de las aportaciones de la cooperación internacional. Lo anterior lo convierte en un ámbito poco propicio, por indefinido y complejo, para la puesta en práctica de políticas. Con todo, a día de hoy prácticamente todas las secretarías de los consejos de ministros sectoriales del SICA cuentan con una agenda de acción política regional. Algunas de ellas incluso están dando lugar a normas reguladoras que podrían hacernos pensar en la posibilidad de una verdadera agenda de integración positiva, lo cual constituye un caso infrecuente en la integración latinoamericana.

En este trabajo analizamos las políticas regionales que se han puesto en marcha en estos últimos años en el marco del SICA, con objeto de determinar si existe una metodología específica de políticas regionales que permita medir su factibilidad y validez como instrumentos de desarrollo para la región y su significado dentro de los procesos de integración. Utilizamos como marco metodológico un enfoque de diseño institucional basado en la sistematización de categorías a partir de los modos de las políticas de la Unión Europea, según la tipología de Helen Wallace (Wallace, 2005: 49-93). No se trata de un análisis comparativo, pues consideramos que, en términos generales, resulta inapropiado realizar ejercicios comparativos procedentes de la extrapolación de distintos modelos de integración, al no existir dos realidades regionales idénticas. Lo que hacemos es utilizar como referencia algunas de las categorías del modelo europeo, precisamente la de aquellas que se adaptan mejor a los marcos centroamericanos. La ausencia de otras referencias en materia de políticas públicas de la integración y las similitudes que presentan los elementos sustantivos de dichas categorías (objetivos de las políticas, gobernanza compleja y multinivel, ciclo de las políticas, actores participantes, intereses, etc.), nos permiten sacar a relucir algunas de las peculiaridades más notorias que se están manifestando a la hora de implementar la agenda centroamericana de políticas regionales, dejando para próximos trabajos un análisis más exhaustivo acerca de las razones jurídicas, institucionales y políticas de las mismas.

La estructura del documento consta de tres secciones. En primer lugar, analizamos los términos del debate en materia de políticas regionales, señalando el cuestionamiento que se cierne sobre la naturaleza de las acciones de políticas en los proyectos integracionistas, procedente de su singularidad con respecto a las políticas públicas tradicionales. A continuación, en el tercer epígrafe, describimos el marco institucional de las acciones de política regional que se han puesto en marcha en el SICA, como elemento condicionante del proceso. El cuarto epígrafe identifica y sistematiza cada una de las políticas a partir del modelo de Wallace para la Unión Europea y del establecimiento de una serie de categorías en las distintas fases de una política pública: la identificación del problema, el diseño, la toma de decisiones, la ejecución y la evaluación. El documento finaliza con las conclusiones de rigor.

\section{El debate sobre las políticas regionales de la integración}

El primer aspecto destacado de las políticas en el marco de la integración regional es su singularidad, relacionada con su condición de sistema político propio que opera a nivel transnacional, distinto pero complementario al del Estado-nación. En los últimos años, existe una abundante literatura que pone de relieve las dificultades para la puesta en práctica de políticas públicas en espacios transnacionales pese a la creciente interdependencia sistémica, ya sea desde procesos de transferencia, difusión o convergencia de políticas (Gilardi, 2012; Holzinger y Knill, 2005; Knill, 2005; Weyland, 2007; Simmons y Elkins, 2004; Drezner, 200I). En el caso de las políticas situadas en el ámbito de la integración regional, el funcionamiento óptimo de este tipo de procesos es más complejo, si cabe. Requiere un marco integracionista avanzado, con instrumentos jurídicos, políticos e institucionales que provean los mecanismos de implementación necesarios y su coordinación con los del Estado subsidiario encargado de ejecutar dichas políticas. Esta peculiaridad hace difícil su comparación con otros procesos de políticas públicas fuera de los paradigmas de la integración. Salvo en el caso europeo, los distintos proyectos integracionistas que han aflorado en las dos últimas décadas por todo el mundo apenas han logrado articular una agenda de políticas de esta naturaleza. No es una tarea fácil. El férreo intergubernamentalismo predominante se constituye en un importante factor obstaculizador. Este hecho se percibe a priori en aspectos como la unanimidad en el proceso 
de toma de decisiones, en la limitación del rol de las instituciones de la integración al de meros promotores y facilitadores de las políticas y en las dificultades para la articulación de las agendas regional y nacionales, que dificulta la adopción e implementación de dichas políticas por parte de los ministerios o de las agencias nacionales.

A diferencia de lo que ocurre para los casos de las políticas públicas globales o de la integración europea, donde existe un rico y nutrido catálogo de trabajos como consecuencia de su amplia trayectoria (Scharpf, 2000; Wallace, Wallace y Pollack, 2005; Majone, 2005; Hix, 2005), el estudio de las políticas surgidas de los marcos institucionales de la integración o del regionalismo latinoamericano es un campo todavía explorado insuficientemente. Los estudios realizados se han centrado en el impacto de las políticas más reconocibles, es decir, las de libre comercio, las de transferencias condicionadas o el de las políticas sociales (Riggirozzi, 20I4 y 20I5).

Algunas de ellas concluyen la necesidad de alcanzar mayores niveles de compromiso por parte de los gobiernos y en la reducción de las asimetrías para alcanzar entornos más eficientes (Giordano, Lanzafame y Meyer-Stamer, 2005). De manera complementaria, otros diagnósticos vinculados a la calidad de las políticas latinoamericanas convergen con los argumentos clásicos, relacionados con la efectividad del sistema político y la calidad de las instituciones. Desde un enfoque más político, otros trabajos destacados señalan como claves una serie de variables políticas e institucionales, tales como la capacidad de formulación de políticas por parte de las cámaras legislativas, la institucionalización del sistema de partidos, la independencia judicial y la calidad burocrática (Franco y Scartascini, 2014; Stein et al., 2006 y 2008; Scartascini, Stein y Tommassi, 2013). En la misma línea, podrían situarse los indicadores de gobernanza de Kaufmann, Kray and Matruzzi (20I0), que miden la eficacia del gobierno, la calidad regulatoria, el control de la corrupción o el imperio de la ley. Unos y otros destacan los rezagos de América Latina con respecto a otras regiones.

Aunque todos estos enfoques aportan herramientas para el análisis, su racionalidad no se ajusta adecuadamente al marco institucional y de gobernanza de la integración regional, donde aparecen otras categorías diferenciadas. En este caso, el análisis debe contemplar, por ejemplo, la existencia de marcos regulatorios transnacionales, de una gobernanza multinivel que opera con mecanismos de imposición, coordinación y cooperación y, a la postre, que tengan en cuenta la conducta de un conjunto de actores más heterogéneos, reacios y distantes con respecto a los centros de decisión y de poder, cuya legitimación democrática se lleva a cabo de manera indirecta en el mejor de los casos. En definitiva, se trata de un marco más complejo para la formulación de políticas y menos practicado, en el que el peso de la gobernanza regional y el papel de las instituciones regionales en la adopción de decisiones independientes de los Estados miembros resulta clave (Sandholtz y Stone Sweet, 1998).

Pero ninguno de los proyectos integracionistas latinoamericanos ha desarrollado las capacidades institucionales necesarias para poner en práctica un sistema político efectivo que estuviese en disposición de producir bienes públicos regionales. Estos proyectos se esforzaron en la creación y ampliación de mercados y en el desarrollo de instituciones, pero no llegaron a establecer verdaderas agendas de políticas comunes.

El objetivo prioritario fue la integración negativa, es decir, la eliminación de aranceles y restricciones al comercio bajo un esquema de gobernanza intergubernamental, y no tanto la acción de integración positiva que pone en manos de las instituciones regionales la capacidad de establecer regulaciones y acciones correctoras sobre el nuevo mercado regional que se establece (Tinbergen, 1957; Scharpf, 2000; Sanahuja, 2007). No obstante, en el caso centroamericano (SICA), la presión de la naturaleza multidimensional de las instituciones regionales ha terminado por promover, de una u otra forma, una cartera de acciones de política regional en casi todos los proyectos integracionistas, en un ejemplo de la influencia que la construcción institucional ejerce sobre los procesos de políticas (Pierson, 2000) y específicamente sobre los proyectos integracionistas (Armstrong y Bulmer, 1998; Sandholtz y Stone Sweet, 1998). En la última década, desde el SICA se ha conseguido poner en marcha, con el liderazgo de los Consejos de Ministros y el apoyo de la cooperación internacional, un conjunto de acciones de política regional cuyos mayores obstáculos se encuentran, precisamente, en la indefinición del sistema político 
regional. Esta vulnerabilidad se compensa con un alto nivel de consenso acerca de su pertinencia y con la disponibilidad de los stakeholders.

Su consideración de políticas regionales, no obstante, se puede poner en entredicho debido al cuestionamiento de la existencia de un gobierno regional con legitimidad y capacidad coercitiva que actúe como rector del proceso. Las definiciones clásicas de política pública tienden a considerar como tal aquellas iniciativas que surgían de en la actividad de las instituciones de gobierno casi exclusivamente (Meny y Thoenig, 1989;

Dye, 1987). Sin embargo, otros enfoques más genéricos definen una política pública como un conjunto de decisiones y actividades ligadas a la solución de un problema colectivo (Dunn, 198I), haciendo énfasis en el objeto, es decir, en la satisfacción de una demanda, de una necesidad o de una oportunidad de intervención pública, y no tanto en el sujeto institucional que la lleva a cabo. Una visión actualizada de este mismo enfoque define políticas púbicas como las "acciones y decisiones llevadas a cabo por diversos actores con la finalidad de resolver un problema definido políticamente como público" (Subirats, Knoepfel, Larrue, Varone, 2007, p. 38). Se entienden las políticas públicas, por tanto, como una construcción social plural que requiere no sólo la participación de las instituciones públicas, sino también de otros actores privados o públicos supranacionales, incluso superando las fronteras del Estado-nación.

El marco jurídico de la integración centroamericana cuenta con mecanismos jurídicos vinculantes, los reglamentos, que permiten la incorporación de las políticas regionales a los procesos nacionales. Pero su elaboración por parte de los distintos consejos de ministros del SICA que pusieron en práctica agendas de políticas sectoriales no ha sido lo habitual, lo que nos lleva a pensar que no existe el suficiente grado de consenso o de voluntad para su puesta en práctica efectiva. Sin este instrumento, la política queda como una iniciativa de coordinación entre países, dejando su implementación a factores coyunturales. No obstante, la existencia de una estructura política e institucional y la apertura de los procesos a la participación de los actores interesados justificarían su consideración de políticas públicas. Una cuestión diferente sería la denominación de sus niveles de institucionalización, o si el diseño institucional y los mecanismos de gobernanza disponibles suponen un factor facilitador u obstaculizador para su puesta en práctica efectiva.

El problema fundamental, sin embargo, es que su implementación carece de una metodología que permita hacer factible esta posibilidad. Esta es la razón que justifica la necesidad de buscar métodos de análisis específicos que contribuyan a identificarlos y difundirlos entre los policy makers.

\section{El marco de las políticas regionales del SICA}

El Sistema de la integración Centroamericana (SICA) se encuadra dentro de los procesos del Nuevo Regionalismo que se ponen en marcha en la década de los noventa del pasado siglo en América Latina bajo la modalidad del regionalismo abierto.

Tiene su origen, sin embargo, en la segunda ola del regionalismo latinoamericano que se desarrolla desde la década de los cincuenta, constituyéndose en uno de los procesos históricos latinoamericanos. Este primer regionalismo cerrado se construyó en torno al Mercado Común Centroamericano (MCCA), suscrito en 1960 el marco de la Organización de Estados Centroamericanos (ODECA) con la participación de Costa Rica, El Salvador, Guatemala, Honduras y Nicaragua. El proceso evolucionó satisfactoriamente hasta la segunda mitad de los setenta, muy afectado por la crisis internacional, hasta desembocar en el conflicto armado que vivió Centroamérica en la década de los ochenta.

La paz de Esquipulas ejerció de catalizador en la reactivación del proceso, que tuvo lugar en diciembre de 199| con la creación del Sistema de Integración Centroamericano (SICA) a través del Protocolo de Tegucigalpa, que subroga a la ODECA. En 1993 los países miembros reactivan la integración económica mediante el Protocolo de 
Guatemala, que conforma una unión aduanera hoy vigente aunque de forma incompleta. Posteriormente, en 1995 se firma el Tratado de Integración Social y el Tratado Marco de Seguridad Democrática. Durante ese proceso se han ido incorporando Belice, Panamá y, más recientemente, la República Dominicana.

El SICA se define políticamente por un marco jurídico político de inspiración comunitaria que, sin embargo, ha configurado una estructura institucional de marcado carácter intergubernamental (Salazar, 20I4; Santos, 20I4). La contradicción que late en su seno ha sido objeto de crítica permanente y objeto de una propuesta de reforma institucional casi permanente. Cuenta con cinco subsistemas (político, económico y comercial, social, cultural y educativo y ambiental), que le otorgan un carácter multidimensional, muestra del compromiso con el desarrollo que supuso el proyecto inicial de Esquipulas. Sin embargo, la adopción del regionalismo abierto cambió el curso del proceso, pasando a centrarse en la agenda económica y comercial.

Tras los distintos intentos fallidos de reforma, el "Relanzamiento de la Integración Centroamericana", un programa impulsado a finales de 2010, consiguió ordenar la agenda y retomar la dinámica de formulación de políticas en torno a cinco ejes, confirmando de esta manera la apuesta por la multidimensionalidad y por los objetivos de desarrollo. Estos ejes fueron el de seguridad democrática, integración económica, integración social, prevención y mitigación de desastres naturales y de efectos del cambio climático y fortalecimiento institucional. En este marco se han conseguido poner en marcha un conjunto de iniciativas de política pública regional, algunas de gran relevancia internacional como la Estrategia de Seguridad de Centroamérica, y de impacto, como la compra conjunta de medicamentos. Pero hay otras desarrollándose en la actualidad, todas ellas posibles gracias a la confluencia de varios procesos: un proceso de gobernanza regional bajo el liderazgo de las instituciones de la integración; la consolidación de una serie de coaliciones entre distintos grupos de interés en el seno de una sociedad civil regional incipiente; y el apoyo de la cooperación internacional, que ha trasladado el grueso de sus recursos destinados a la región desde el ámbito bilateral al regional.

De acuerdo a los patrones institucionales del SICA, corresponde a los distintos Consejos de Ministros sectoriales la materialización de las políticas, que se gestan en el marco de sus secretarías respectivas. Una vez definidas, con la mediación del Comité Ejecutivo ${ }^{36}$, se elevan a la aprobación de la Reunión de Presidentes, que lo hace por consenso. Sin embargo, esta decisión presidencial no conlleva obligatoriedad ni juridicidad subsidiaria. Esta última sólo se establece mediante la aprobación de un reglamento en el seno del Consejo de Ministros sectorial correspondiente, firmada por los ministros representantes de cada país que esté dispuesto a implementar la política.

En este caso, la publicación en el diario oficial nacional incorpora la política a los marcos jurídicos nacionales. Sin embargo, el SICA no tiene definidas competencias específicas en materia de política, que quedan bajo el control y seguimiento de los gobiernos respectivos una vez han sido incorporados a su sistema político y jurídico.

Tampoco dispone de instrumentos financieros que permitan su financiación directa. Hasta entonces, las acciones de políticas aprobadas pueden ser implementadas por iniciativa de las distintas secretarías de los consejos de ministros sectoriales del SICA, con la colaboración y el compromiso de los gobiernos, pero no de forma vinculante. Por lo general, este proceso se lleva a cabo en los casos en los que la política cuenta con un mínimo de financiación para operar.

En este caso, el papel de articulación y liderazgo que están jugando los distintos órganos e instituciones del SICA es fundamental, aunque insuficiente. El proceso de fortalecimiento institucional llevado a cabo a lo largo de estos años ha propiciado el reforzamiento técnico y la continuidad de los cuerpos funcionariales, así como una mayor

\footnotetext{
${ }^{36}$ En un proceso que es irregular porque no siempre las propuestas de política pasan a través del Comité Ejecutivo ni existe una normativa específica que precise cuando debe hacer y en qué condiciones.
} 
eficacia de los procedimientos burocráticos. Por otro lado, la creación de espacios de participación para la sociedad civil permitió la construcción de un proceso de concertación de distintos sectores de la sociedad civil en torno a los intereses regionales, cuya evolución, no obstante se está viendo obstaculizada por las debilidades institucionales y por la desconfianza con respecto a la voluntad política de los gobiernos. No obstante, estos grupos suelen estar muy presentes y activos en el proceso de diseño de las políticas, contrarrestando el déficit de legitimidad que pudiera recaer sobre los distintos procesos.

\section{4. los modos de las políticas del SICA}

En esta sección, procedemos a llevar a cabo nuestro análisis sobre el conjunto de acciones políticas del SICA. Metodológicamente, se basa en la sistematización de una serie de categorías establecidas a partir de dos elementos: la referencia al modelo europeo, por ser éste el que ha desarrollado un proceso de este tipo, profundo y contrastado; y el ciclo de las políticas públicas, que nos permite analizar cómo se responde a las distintas preguntas formuladas en un proceso de definición de una política pública. La información procede de los distintos trabajos acumulados a lo largo de estos años de experiencia en la integración centroamericana, de los documentos oficiales de las distintas políticas analizadas y de las opiniones de distintos actores cualificados.

\section{I. Los modos de las políticas de la Unión Europea (UE)}

Tomamos como punto de partida la propuesta de modos de política de la Unión Europea catalogadas por Helen Wallace (Wallace, 2005: 49-93). No se trata de usarlo como modelo comparativo, sino de tomar los elementos que nos ofrece ya categorizados sobre una lógica similar, mucho más desarrollada, para aplicarlos sobre nuestro análisis.

\begin{tabular}{|c|c|c|c|c|}
\hline $\begin{array}{l}\text { MODO DE EJECUCIÓN } \\
\text { DE LA POLIITICA }\end{array}$ & $\begin{array}{c}\text { GRADO DE } \\
\text { PARTICIPACIÓN DE } \\
\text { LAS INSTITUCIONES } \\
\text { SUPRANACIONALES }\end{array}$ & $\begin{array}{c}\text { GRADO DE } \\
\text { PARTICIPACIÓN DE } \\
\text { OTROS ACTORES Y } \\
\text { GRUPOS DE INTERÉS }\end{array}$ & $\begin{array}{c}\text { TIPO DE } \\
\text { COMPETENCIAS }\end{array}$ & $\begin{array}{l}\text { DEFANICIÓN DE LA } \\
\text { FINANCIACIÓN }\end{array}$ \\
\hline COMUNITARIA & $\begin{array}{l}\text { Proceso institucional } \\
\text { jerárquico y centralizado }\end{array}$ & $\begin{array}{c}\text { Los grupos } \\
\text { potencialmente afectados } \\
\text { por la política } \\
\text { (stakeholders) son } \\
\text { integrados en el proceso } \\
\text { decisorio (se alinean a } \\
\text { nivel transnacional) } \\
\end{array}$ & Exclusivas & Financiación comunitaria \\
\hline REGULATORIA & $\begin{array}{l}\text { Proceso institucional no } \\
\text { totalmente centralizado }\end{array}$ & $\begin{array}{l}\text { Elevada participación de } \\
\quad \text { los stakeholders } \\
\text { (especialmente empresas } \\
\text { y lobbies) y de expertos. }\end{array}$ & Compartidas & $\begin{array}{l}\text { Cofinanciación UEy } \\
\text { Estados nacionales }\end{array}$ \\
\hline DISTRIBUTIVA & $\begin{array}{l}\text { Proceso institucional } \\
\text { multinivel }\end{array}$ & $\begin{array}{c}\text { Participación moderada } \\
\text { de stakeholders y muy } \\
\text { elevada de las } \\
\text { autoridades } \\
\end{array}$ & Compartidas & $\begin{array}{l}\text { Cofinanciación UEy } \\
\text { Estados nacionales }\end{array}$ \\
\hline COORDINADA & $\begin{array}{l}\text { Proceso institucional } \\
\text { descentralizado }\end{array}$ & $\begin{array}{c}\text { Elevada participación de } \\
\text { stakeholders, sobre todo } \\
\text { de expertos } \\
\text { independientes }\end{array}$ & Coordinadas & $\begin{array}{l}\text { Cofinanciación UEy } \\
\text { Estados nacionales }\end{array}$ \\
\hline TRANSGUBERNAMENTAL & $\begin{array}{l}\text { Proceso institucional muy } \\
\text { descentralizado }\end{array}$ & $\begin{array}{l}\text { Elevada participación de } \\
\text { los policy-makers } \\
\text { nacionales }\end{array}$ & Nacional & Financiación nacional \\
\hline
\end{tabular}

La propuesta de Wallace permite observar como la integración proporciona espacios para la definición de políticas regionales con distintos niveles de competencias, ofreciendo distintos escenarios que van desde lo supranacional a lo meramente nacional. Contribuye, de esta manera, a matizar el mito de la necesidad de un marco supranacional 
que se cierne sobre los procesos latinoamericanos de integración, ofreciendo a cambio una gama de objetivos y herramientas metodológicas que permiten superar esta dialéctica.

La supranacionalidad sólo se vislumbra en las políticas de tipo comunitario exclusivamente, debido al carácter exclusivo de sus competencias, mientras que los modos regulatorio, distributivo y coordinado alcanzan distintos niveles de descentralización de competencias. Los modos regulatorio y distributivo requieren algún tipo de supranacionalidad, debido a que exige competencias compartidas y mecanismos de control y coerción independientes de los gobiernos nacionales. Por lo general, la competencia regional se vincula al control del cumplimiento de los reglamentos y los términos de la distribución de recursos que tiene lugar en las políticas distributivas. En este último caso, es importante también su labor de articulación y cohesión de los distintos actores que participan del proceso multinivel que tiene lugar. En las políticas coordinadas, por su parte, la participación de las instituciones supranacionales se diluye todavía más, restringiendo sus competencias a la coordinación, concertación de medios y esfuerzos, etc., principalmente con los gobiernos nacionales. Finalmente, en el modo transgubernamental, las instituciones no tienen competencias, de ahí que su papel se limite a la colaboración.

Uno de los aspectos más llamativos en la clasificación de Wallace es la notoria participación de los actores vinculados a los grupos de interés en todos los modos. La definición de políticas regionales contribuye, sin duda, a la definición de una gobernanza en la que las instituciones regionales tienen mayor protagonismo. En el caso del modo comunitario, la institución regional se convierte en la única interlocutora, sustituyendo a los estados en el monopolio de la interlocución con los actores de la sociedad civil, que deben coaligarse a nivel transnacional. Los modos regulatorio y coordinado ofrecen también incentivos para la participación de actores expertos e independientes.

\subsection{Una sistematización de las políticas del SICA}

El catálogo de políticas activadas en el SICA es todavía escaso, especialmente si tenemos en cuenta la extensa trayectoria de la integración centroamericana. Es también un proceso reciente que sugiere la falta de consolidación en muchas de las iniciativas. Con la excepción de las políticas de la integración económica y la de pesca, se localizan la mayoría a partir de 2010, vinculadas por tanto a la estrategia de Relanzamiento. Esto nos da una idea de la evolución que ha seguido el proceso y de la estrecha vinculación entre su dinamismo y las decisiones estratégicas tomadas por actores no directamente relacionados con la demanda de las políticas específicamente, como la decisión de los presidentes o el cambio de estrategia de una parte importante de la cooperación internacional con la región (la europea y la española), que ha trasladado el eje de su esfuerzo a los espacios regionales.

En estos momentos, en el SICA se registra pueden distinguir dos grupos de políticas, las que se encuentran en curso y las que se encuentran aprobadas por la Reunión de Presidentes pero no cuentan con la financiación necesaria para su puesta en marcha.

Este es un primer factor determinante que nos informa acerca de la calidad del proceso. No obstante, la presencia de la cooperación internacional es una oportunidad para paliar en alguna medida esta deficiencia.

Dentro de esta primera diferenciación, de las políticas que se hallan en curso, se pueden distinguir dos modos de ejecución: el regulatorio y el de coordinación, aunque existen otras que podrían aspirar a convertirse en políticas distributivas. El modo regulatorio se caracteriza por dictar una norma reguladora y limitante que afecta al comportamiento económico de un sector determinado. En el caso centroamericano, las competencias no están definidas, aunque de facto son compartidas, puesto que la norma reguladora se concreta en el Consejo de 
Ministros regional, apoyándose en los grupos de interés y las empresas, pero las instituciones regionales carecen de competencias una vez han sido insertadas en los sistemas legales nacionales, por lo que el grado de participación en el proceso se queda en el diseño de la política.

Cuadro $n^{\circ}$ 2. Políticas regionales del SICA en curso. Elaboración propia

\begin{tabular}{|c|c|c|c|c|c|c|c|}
\hline POLÍTICA & AÑO & $\begin{array}{l}\text { MODO DE } \\
\text { EJECUCIÓN }\end{array}$ & $\begin{array}{c}\text { GRADO DE } \\
\text { PARTICIPACIÓN DE } \\
\text { LAS INSTITUCIONES } \\
\text { SUPRANACIONALES }\end{array}$ & $\begin{array}{c}\text { GRADO DE } \\
\text { PARTICIPACIÓN DE } \\
\text { OTROS ACTORES Y } \\
\text { GRUPOS DE INTERÉS }\end{array}$ & COMPETENCIAS & FNANCIACIÓN & SITUACIÓN \\
\hline $\begin{array}{c}\text { Mercado regional } \\
\text { (Reglamentos de } \\
\text { armonización aduanera, } \\
\text { procedimientos } \\
\text { armonización arancelaria, } \\
\text { códigos aduaneros, etc.) }\end{array}$ & Desde 1993 & Regulatorio & $\begin{array}{l}\text { Proceso institucional no } \\
\text { totalmente centralizado }\end{array}$ & $\begin{array}{l}\text { Participación limitada de } \\
\text { las empresas y lobbies. }\end{array}$ & $\begin{array}{l}\text { No definidas formalmente, } \\
\text { pero de facto compartidas }\end{array}$ & $\begin{array}{l}\text { Cofinanciación SICA (a } \\
\text { través de la } \\
\text { cooperación } \\
\text { internacional) y los } \\
\text { Estados nacionales }\end{array}$ & $\begin{array}{l}\text { En curso, sin información } \\
\text { sobre los niveles de } \\
\text { implementación }\end{array}$ \\
\hline $\begin{array}{l}\text { Política de integración de } \\
\text { pesca y acuicultura }\end{array}$ & 2005 & Regulatorio & $\begin{array}{l}\text { Proceso institucional no } \\
\text { totalmente centralizado }\end{array}$ & $\begin{array}{c}\text { Participación de los } \\
\text { empresas, lobbies y } \\
\text { expertos. }\end{array}$ & $\begin{array}{l}\text { No definidas formalmente, } \\
\text { pero de facto compartidas }\end{array}$ & $\begin{array}{c}\text { Cofinanciación SICA (a } \\
\text { través de la } \\
\text { cooperación } \\
\text { internacional) y los } \\
\text { Estados nacionales }\end{array}$ & $\begin{array}{l}\text { En curso, sin información } \\
\text { sobre los niveles de } \\
\text { implementación }\end{array}$ \\
\hline $\begin{array}{l}\text { Negociación conjunta de } \\
\text { la compra de } \\
\text { medicamentos para los } \\
\text { sistemas de salud }\end{array}$ & 2010 & Regulatorio & $\begin{array}{l}\text { Proceso institucional no } \\
\text { totalmente centralizado }\end{array}$ & $\begin{array}{c}\text { Participación de los } \\
\text { empresas, lobbies y } \\
\text { expertos. }\end{array}$ & $\begin{array}{l}\text { No definidas formalmente, } \\
\text { pero de facto compartidas }\end{array}$ & $\begin{array}{l}\text { Cofinanciación SICA (a } \\
\text { través de la } \\
\text { cooperación } \\
\text { internacional) y los } \\
\text { Estados nacionales }\end{array}$ & $\begin{array}{l}\text { En curso, sin información } \\
\text { sobre los niveles de } \\
\text { implementación }\end{array}$ \\
\hline $\begin{array}{c}\text { Estrategia } \\
\text { Centroamericana de } \\
\text { Desarrollo Rural Territorial } \\
\text { (ECADERT) }\end{array}$ & 2010 & Coordinada & $\begin{array}{l}\text { Proceso institucional } \\
\text { descentralizado }\end{array}$ & $\begin{array}{c}\text { Participación de los } \\
\text { empresas, lobbies y } \\
\text { expertos. }\end{array}$ & $\begin{array}{l}\text { No definidas formalmente, } \\
\text { pero de facto coordinadas }\end{array}$ & $\begin{array}{l}\text { Financiación del SICA } \\
\text { (con fondos de la } \\
\text { cooperación } \\
\text { internacinoal) }\end{array}$ & $\begin{array}{l}\text { En curso, sin información } \\
\text { sobre los niveles de } \\
\text { implementación }\end{array}$ \\
\hline $\begin{array}{l}\text { Estrategia de Seguridad } \\
\text { de Centroamérica (ESCA) }\end{array}$ & 2012 & Coordinada & $\begin{array}{l}\text { Proceso institucional } \\
\text { descentralizado }\end{array}$ & $\begin{array}{c}\text { Participación de los } \\
\text { empresas, lobbies y } \\
\text { expertos. }\end{array}$ & $\begin{array}{l}\text { No definidas formalmente, } \\
\text { pero de facto coordinadas }\end{array}$ & $\begin{array}{l}\text { Financiación del SICA } \\
\text { (con fondos de la } \\
\text { cooperación } \\
\text { internacinoal) }\end{array}$ & $\begin{array}{l}\text { En curso, sin información } \\
\text { sobre los niveles de } \\
\text { implementación }\end{array}$ \\
\hline
\end{tabular}


La participación de otros actores y grupos de interés es limitada, salvo en la política de pesca y acuicultura, donde ha registrado una amplia participación.

Uno de los elementos distintivos de las políticas regulatorias es que son las únicas que cuentan con una financiación compartida entre el SICA y los gobiernos nacionales. EI SICA proporciona financiación procedente de la cooperación internacional, generalmente para actividades relacionadas con el fortalecimiento institucional y otras complementarias, mientras que los gobiernos se encargan de financiar la ejecución en su territorio. Sin embargo, a diferencia del estrecho seguimiento que suele caracterizar a estas políticas en Europa, no se cuenta con sistemas de seguimiento de la información acerca de los niveles de ejecución ni del cumplimiento de los reglamentos por parte de los países. Ello es debido a que no se han definido competencias ni instrumentos financieros, por lo que la ejecución se supedita a la búsqueda de fondos y el seguimiento al interés del financiador. Si tenemos en cuenta que incluye las políticas de regulación del mercado regional, fundamentales para el proceso, convenimos en que se trata de una importante debilidad. La apreciación, no obstante, por parte de los actores involucrados es muy favorable en el caso de los beneficios de los reglamentos de acuicultura y pesca, así como del proceso de negociación conjunta de compra de medicamentos. De hecho, en este último caso, se registra una ampliación del volumen de compras, y de los países que se han incorporado a la política.

En cuanto al modo de coordinación, reúne a un conjunto de políticas promovidas por las instituciones regionales que han despertado el interés por parte de los gobiernos, sin llegar a buscar compromisos vinculantes, por lo que su ejecución es contingente. El objetivo es la armonización de las distintas políticas nacionales, incrementando de esta manera la interdependencia. Por lo general, no existen condiciones para desarrollar un proceso más profundo. Aquí, el papel de las instituciones es coordinar los esfuerzos con los gobiernos y los distintos actores participantes, por tanto el proceso de participación es bastante descentralizado. Estas competencias de coordinación parten de una propuesta estratégica común a la que los países pueden sumarse e incorporar a su marco político, jurídico e institucional.

La participación de los actores ha sido amplia en el caso de la Estrategia Centroamericana de Desarrollo Rural Territorial (ECADERT), donde se ha llegado a configurar una institucionalidad nacional y subnacional participada por los propios actores de los territorios. En la estrategia de seguridad ha sido mucho más reducida, con un liderazgo muy centrado en las cancillerías de los países miembros, aunque también ha contado con la participación de los cuerpos profesionales involucrados.

Una de las características de estas políticas es la precariedad de la financiación, debido a que procede únicamente de los fondos de la cooperación aportados por el SICA. En este caso, los gobiernos sólo participan asumiendo los costes de la incorporación de los distintos procesos administrativos y burocráticos. La dependencia de los fondos de la cooperación conlleva también riesgos importantes en todo el proceso de definición de la política, al incorporar un actor con intereses diferenciados.

El segundo grupo de políticas, es decir, el de aquellas políticas aprobadas por los presidentes centroamericanos que han tenido un grado de implementación muy reducido o parcial sin poner en marcha los mecanismos institucionales previstos en el diseño de la política, es mucho más amplio y variado, hasta alcanzar el número de once (II). El principal motivo que impide su ejecución es la falta de financiación y el insuficiente interés mostrado por los países miembros, por diversos motivos. 
Cuadro $n^{\circ}$ 3. Propuestas de políticas regionales del SICA de implementación parcial o reducido Elaboración propia

\begin{tabular}{|c|c|c|c|}
\hline $\begin{array}{l}\text { POLÍTICA O } \\
\text { ESTRATEGIA }\end{array}$ & ÓRGANO RECTOR & ÓRGANO GESTOR & $\begin{array}{l}\text { AÑO DE } \\
\text { INICIO }\end{array}$ \\
\hline $\begin{array}{l}\text { Política Agrícola } \\
\text { Centroamericana }\end{array}$ & $\begin{array}{l}\text { Consejo de Ministros de } \\
\text { Agricultura, Pesca y } \\
\text { Acuicultura }\end{array}$ & $\begin{array}{l}\text { Secretaría del Consejo } \\
\text { Agropecuario } \\
\text { Centroamericano } \\
\text { (SECAC) }\end{array}$ & 2008 \\
\hline $\begin{array}{c}\text { Estrategia Regional } \\
\text { Agroambiental y de Salud } \\
\text { (ERAS) }\end{array}$ & $\begin{array}{l}\text { Consejo de Ministros de } \\
\text { Ambiente y Desarrollo }\end{array}$ & $\begin{array}{l}\text { Secretaría del Consejo } \\
\text { Centroamericano de } \\
\text { Ambiente y Desarrollo } \\
\text { (CCAD) }\end{array}$ & 2008 \\
\hline $\begin{array}{l}\text { Agenda Estratégica Social } \\
\text { de Centroamérica }\end{array}$ & $\begin{array}{l}\text { Consejo de Ministros de } \\
\text { Integración Social }\end{array}$ & $\begin{array}{c}\text { Secretaría de la } \\
\text { Integración Social } \\
\text { Centroamericana } \\
\text { (SISCA) }\end{array}$ & 2008 \\
\hline $\begin{array}{l}\text { Agenda Estratégica y Plan } \\
\text { de Salud de Centroamérica } \\
\text { y República Dominicana }\end{array}$ & Consejo de Ministros de Salud & $\begin{array}{c}\text { Secretaría del Consejo } \\
\text { de Ministros de Salud } \\
\text { CA (SECOMISCA) }\end{array}$ & 2009 \\
\hline $\begin{array}{c}\text { Estrategia Centroamericana } \\
\text { de Vivienda y } \\
\text { Asentamientos Humanos } \\
\text { (ECVAH) }\end{array}$ & $\begin{array}{l}\text { Consejo de Ministros de } \\
\text { Vivienda }\end{array}$ & $\begin{array}{c}\text { Consejo } \\
\text { Centroamericano de } \\
\text { Vivienda y } \\
\text { Asentamientos Humanos } \\
\text { (CCVAH) }\end{array}$ & 2009 \\
\hline $\begin{array}{c}\text { Estrategia Regional de } \\
\text { Cambio Climático }\end{array}$ & $\begin{array}{l}\text { Consejo de Ministros de } \\
\text { Ambiente y Desarrollo }\end{array}$ & CCAD & 2010 \\
\hline $\begin{array}{c}\text { Estrategia Regional de } \\
\text { Cambio Climático (PCGIR) }\end{array}$ & $\begin{array}{c}\text { Consejo de Ministros de } \\
\text { Seguridad, Justicia y } \\
\text { Gobernación }\end{array}$ & $\begin{array}{l}\text { Centro de Coordinación } \\
\text { para la Prevención de } \\
\text { Desastres Naturales en } \\
\text { América Central } \\
\text { (CEPREDENAC) }\end{array}$ & 2010 \\
\hline $\begin{array}{l}\text { Política Cultural de } \\
\text { Integración } \\
\text { Centroamericana }\end{array}$ & $\begin{array}{l}\text { Consejo de Ministros de } \\
\text { Cultura }\end{array}$ & $\begin{array}{c}\text { Secretaría de la } \\
\text { Coordinación Educativa } \\
\text { y Cultura de } \\
\text { Centroamérica } \\
\text { (SECECC) }\end{array}$ & 2012 \\
\hline $\begin{array}{l}\text { Política Educativa } \\
\text { Centroamericana }\end{array}$ & $\begin{array}{c}\text { Consejo de Ministros de } \\
\text { Educación }\end{array}$ & SECECC & 2013 \\
\hline $\begin{array}{l}\text { Política Regional de } \\
\text { Igualdad y Equidad de } \\
\text { Género (PRIEG) }\end{array}$ & $\begin{array}{c}\text { Consejo de Ministras de la } \\
\text { Mujer }\end{array}$ & $\begin{array}{l}\text { Secretaría del Consejo } \\
\text { de Ministras de la Mujer } \\
\text { de Centroamérica } \\
\text { (SECOMMCA) }\end{array}$ & 2013 \\
\hline $\begin{array}{c}\text { Política de Seguridad } \\
\text { Alimentaria y Nutricional de } \\
\text { Centroamérica y República } \\
\text { Dominicana 2012-2032 }\end{array}$ & Consejo de Ministros de Salud & $\begin{array}{c}\text { Instituto de Nutrición de } \\
\text { Centroamérica y Panamá } \\
\text { (INCAP) }\end{array}$ & 2013 \\
\hline
\end{tabular}

Como se observa en el cuadro, la mayoría de estas políticas han sido formuladas en fechas recientes, aunque la demora en su ejecución las arriesga a hacer caer en el olvido, como ocurrió en otras ocasiones. Enfrentan temas de gran relevancia social para la región, revelando el potencial de la integración como marco del desarrollo, al tiempo que, como hecho sintomático, encuentran dificultades para su puesta en práctica en este marco.

Una variable que podría explicar los limitados avances en la ejecución de algunas de estas políticas es su origen y la fundamentación de su pertinencia. No todas las políticas señaladas en el cuadro reunían los consensos necesarios ni los tenían avales suficientes para iniciar el proceso de las instituciones nacionales del sector. O no contaban con aval formal o informal de las instituciones rectoras de la integración en cada país, ya sean los Ministerios de Relaciones Exteriores las 
oficinas presidenciales o los Ministerios de la Presidencia. La ausencia de procedimientos explícitos para la aprobación de políticas permite que en el proceso centroamericano de integración prosperen algunas iniciativas sin los debidos respaldos políticos. Normalmente, esta debilidad no impide que las Declaraciones presidenciales avalen ${ }^{37}$ su diseño pero reduce las posibilidades de ejecución posterior.

Al no existir información regular o institucionalizada de sus avances no pueden ser catalogadas en todas las categorías que hemos presentado aquí. No obstante, las fuentes de información disponibles sobre el proceso de formulación, avanzan que el grado de participación de las instituciones regionales y de los grupos de actores ha sido, por lo general, más amplio de lo que es habitual en las políticas de la región. Del mismo modo, de acuerdo a los objetivos especificados en los distintos programas oficiales y a las informaciones procedentes del proceso de formulación, la mayoría de ellas podrían adscribirse al modo de coordinación de políticas. La excepción podría ser la Política Agraria Centroamericana, quien debido a la problemática que registra podría ser objeto de una política de tipo regulatorio que aglutinase, además, a la ECADERT. No obstante, la posibilidad de definir políticas de tipo distributivo que favorezca a determinados grupos o sectores parece todavía lejana.

\section{Conclusiones}

En las páginas anteriores se ha analizado brevemente el contexto de Centroamérica en relación a las políticas regionales que se promueven en el marco de su proceso de integración regional, y se ha sistematizado el conjunto de políticas derivadas del mismo en los años 2002-2014, basándonos en las categorías establecidas por Helen Wallace para la UE y en el modelo clásico del ciclo de las políticas públicas.

Algunas de las acciones de política regional que se desarrollan en el marco del SICA pueden ser consideradas como políticas regionales válidas, debido a que se trata de intervenciones llevadas a cabo por una institución pública de carácter regional que pretende resolver un problema identificado como público con el apoyo de actores interesados de la sociedad civil y otros socios de las instituciones regionales y gubernamentales.

Aplicando las categorías de modos de ejecución de Helen Wallace a las políticas del SICA hemos detectado que las políticas que se están ejecutando responden al modo de coordinación y de regulación, pero aparecen dos debilidades recurrentes en su diseño que comprometen su ejecución y su viabilidad. Estos modos no son explícitos; es decir, el Sistema no contempla estas categorías porque no ha desarrollado suficientemente el concepto de política regional, y por eso diseña con imprecisión. No obstante, en nuestra opinión, la aplicación de estos modos podría mejorar la eficacia de las políticas regionales.

En la integración centroamericana, las competencias en materia de políticas no están definidas, y por tanto su ejecución no es vinculante para los países miembros del SICA, aunque de facto podrían ser asimilables a unas competencias compartidas y coordinadas. La definición de competencias es un factor determinante para la implementación de políticas, puesto que implica la incorporación de una metodología, es decir: el qué, el quién y el cómo se hace la política. En la práctica de procesos con actos normativos débiles como los latinoamericanos, la definición de competencias condiciona el diseño y la ejecución de las políticas regionales.

Encontramos dos defectos en las políticas que comprometen su viabilidad: la falta de distribución de competencias y la ausencia de mecanismos de financiación. Debido a la falta de centralidad

\footnotetext{
${ }^{37}$ En general, estas políticas son recibidas pero no aprobadas en la redacción habitual de las declaraciones de las Cumbres Presidenciales.
} 
de las políticas regionales, en la integración centroamericana las políticas no suelen incorporar los instrumentos de financiación, a menos que un agente externo los provea. En este último caso, se trata de instrumentos cuya naturaleza no es permanente o sostenible.

La actitud de resistencia de los gobiernos parece estar relacionada con la pérdida de soberanía frente a las instituciones regionales así como por la ausencia de incentivos suficientes para dotar al SICA de recursos de origen nacional o de capacidad estratégica de los Estados para apostar por la acción común y crear las condiciones para una integración eficaz. No obstante, nuestro análisis muestra que es posible propiciar políticas regionales bajo el modo de coordinación que admiten la pervivencia de marcos intergubernamentales. En este caso, la armonización de las políticas no sólo permite avanzar en materia de interdependencia, sino que permiten generar condiciones para avanzar a modos de ejecución que aseguren mayor eficacia como el modo distributivo al que podrían aspirar políticas como la ESCA y la ECADERT.

No ocurre lo mismo con el modo regulatorio, que requiere la utilización de instrumentos coercitivos y cierta cesión de soberanía a un órgano que represente los intereses regionales, dado que la norma reguladora afecta al conjunto de los sectores implicados a nivel regional. En este caso, la cesión de soberanía estaría justificada en aras del cumplimiento de la norma. Es decir, se requieren instituciones regionales con competencias para velar por el cumplimiento de los objetivos, de la eficacia y de la eficiencia de la política.

La conclusión final a la que se llega parece confirmar el diagnóstico popular sobre el predominio de la retórica sobre la voluntad política de los gobiernos en materia de integración; de bloquear los verdaderos procesos de interdependencia. Sin embargo, parece ser también un problema de visibilidad y de capacidad. $Y$ desde luego no despeja la incógnita acerca de cuáles son las razones que llevan a los Estados a mantener una retórica integracionista tan costosa que requiere la construcción de todo un entramado institucional. Son precisamente estas instituciones regionales las que han terminado por generar una gobernanza regional bajo su liderazgo, al conseguir aglutinar a coaliciones de actores y grupos con intereses a este nivel, demostrando con ello que existen razones para la integración.

\section{Bibliografía}

ARMSTRONG, K. A. Y BULMER, S. (1998). The governance of the single European Market. Manchester University Press. Manchester

BEST, E. (2006). Regional Integration and (good) regional governance. Are common standars and indicators possible? En DE LOMBAERDE, Ph. (eds.) (2006). Assessment and Measurement of Regional Integration, Pp. 183-214. London: Routledge.

BULL, B. (2005). Aid, Power and Privatization: The Politics of Telecommunication Reform in Central America. Cheltenham: Edward Elgar

BULMER THOMAS, V. (1987). The Political Economy of Central America Since 1920, New York: Cambridge. University Press.

CALDENTEY DEL POZO, P. (2014). Los desafíos estratégicos de la integración centroamericana. Serie Estudios y Perspectivas $n^{\circ}$ I56. Sede Subregional de la CEPAL en México. México: CEPAL.

DE LOMBAERDE, Ph. (eds.) (2006). Assessment and Measurement of Regional Integration. London: Routledge.

DUNN, W. N. (198I). Public Policy Analysis: An Introduction. Englewood Cliff, N. J: Prentice Hall.

DYE, Th. R. (1987). Understanding Public Policy. Prentice Hall.

FRANCO, M. y SCARTASCINI, C. (20I4). The Politics of Policies: Revisiting the Quality of Public Policies and Government Capabilities in Latin America and the Caribbean. Inter- 
American Development Bank Department of Research and Chief Economist POLICY BRIEF. No. IDB-PB-220.

GIORDANO, P., LANZAFAME, F. Y MEYER-STAMER, J. (2005). Asymmetries in Regional Integration and Local Development. Inter-American Development Bank Washington, D.C.

GILARDI, F. (20I2). Transnational Diffusion: Norms, Ideas and Policies. In Handbook of International Relations, ed. Walter Carlsnaes, Thomas Risse and Beth Simmons. Second ed. SAGE Publications.

HOLZINGER, K. y KNILL, C. (2005). Causes and conditions of cross-national policy convergence. Journal of European Public Policy 12 (5): 775-96

HIX, S. (2005). The Political System of the European Union. Second Edition. Palgrave Macmillan.

KAUFMANN, D.; KRAAY, A. y MASTRUZZI, M. (20I0). The Worldwide Governance Indicators: Methodology and Analytical Issues. Draft Policy Research Working Paper. En http://info.worldbank.org/governance/wgi/pdf/WGl.pdf

KNILL, C. (2005). Cross-national policy convergence: concepts, approaches and explanatory factors. Journal of European Public Policy 12:5. October 2005: 764-774. Routledge.

LEHOUCQ, P. (2012). The Politics of Modern Central America: Civil War, Democratization, and Underdevelopment, by Fabrice Lehoucq. Cambridge and New York: Cambridge University Press.

LIEBMAN, J. B. (20I3). Building on Recent Advances in Evidence-Based Policymaking. The Hamilton Project. Brooking Institutions.

MAJONE, G. (2005). Dilemmas of European Integration. The Ambiguities and Pitfalls of Integration by Stealth. Oxford University Press.

MENY, I. Y THOENIG, J. C. ( 1 989). Las políticas públicas. Ariel. Barcelona.

PELLANDRA, A. Y FUENTES, J. A. (201 I). El estado actual en la integración centroamericana.

PIERSON, P. (1996). The Path to European Integration: A Historical Institutionalist Analysis. Comparative Political Studies 1996; 29; 123. Serie Estudios y Perspectivas $n^{\circ} 129$. CEPAL, Sede subregional en México.

RIGGIROZZI, P. (20I4). Regionalism through social policy: Collective action and health diplomacy en South America. Economy and Society 43 (3): $432-454$

(2015). Regionalism, activism and right: New opportunities for health diplomacy in South America. Review of International Studies 4 I (2): 407-428

RUEDA JUNQUERA, F. Y SÁNCHEZ ANCOCHEA, D. (2009). Integración económica en Centroamérica. Retos, oportunidades y escenarios para España. Documento de Trabajo 35/2008. Fundación Alternativas.

RUEDA JUNQUERA, F. (20I4). Regional Integration in Central America. En Sánchez-Ancochea, D. y Martí i Puig, S. (ed.) (2014). Handbook of Central American Governance. Cap. 20, PP. 335-349. Routledge. London y New York, 2014

SANAHUJA, J. A. (2007) Regionalismo e integración en América Latina: balance y perspectivas. Revista de Pensamiento Iberoamericano, $n^{\circ} 0$. Segunda época, enero de 2007, pp. 75106

SÁNCHEZ-ANCOCHEA, D. Y MARTí I PUIG, S. (ed.) (2014). Handbook of Central American Governance. Routledge. London y New York, 2014

SANDHOLTZ, W. Y STONE SWEET, A. (1998). European Integration and Supranational Governance. Oxford University Press.

SANTOS, F. (20I4). Dilemas de la integración centroamericana: las propuestas de reforma institucional del SICA en perspectiva. En Revista Centroamericana de Administración Pública, $n^{\circ}$ 66-67, Actualidades de la Integración Centroamericana: Experiencias y Perspectivas. En http://www.icap.ac.cr/periodicas/index.php/administracionpublica/search/titles?searchPa ge $=2$

SANTOS, F. y POZO, O. (2013). El SICA: diálogos sobre una integración dinámica y singular en América Latina. Colección de estudios centroamericanos $\mathrm{N}^{\circ} 4$. Córdoba: Fundación ETEA para el Desarrollo y la Cooperación 
SCARTASCINI, C.; STEIN, E.; TOMMASI, M. (20I3) Political Institutional, Intertemporal Cooperation, and the Quality of Public Policies. Journal of Applied Economics. Volume 16, Issue I, May 2013, Pages I-32.

SCHARPF, F. (2000). Gobernar en Europa eficaz y democráticamente. Alianza. Madrid.

SCHIFF, M. Y WINTER, L. A. (2003). Integración regional y desarrollo. Banco Mundial y Editorial Alfaomega. Bogotá.

SEGOVIA, A. (2005). Integración real y grupos de poder en América Central: implicaciones para el desarrollo y la democracia de la región, San José: Frederich Ebert Stiftung

SIMMONS, B. A. y ELKINS, Z. (2004). The globalization of liberalization: policy diffusion in the international political economy. American Political Science Review, 98: 17I-I89

STEIN, E., TOMMASI, M., SCARTASCINI, C. Y SPILLER, P. (2006) Policymaking in Latin America How Politics Shapes Policies

STONE SWEET, A.; SANDHOLTZ, W. Y FLIGSTEIN, N. (200I). The Institutionalization of Europe. Oxford University Press. New York.

SUBIRATS, J.; KNOEPFEL, P.; LARRUE, C. Y VARONE, F. (2008). Análisis y gestión de políticas públicas. Ariel. Barcelona.

SUTCLIFFE, S. Y COURT, J. (2005). Evidence-Based Policymaking: What is it? How does it work? What relevance for developing countries? Project: "Evidence-based Policymaking: Lessons from Uk for Developing Countries". En http://www.odi.org/sites/odi.org.uk/files/odi-assets/publications-opinion-files/3683.pdf

TINBERGEN, J. (1954). International Economic Integration. Amsterdam, Elsevier.

WALLACE, H. (2005). An Institutional Anatomy and Five Policy Modes. En WALLACE, H.; WALLACE, W. Y POLLACK, M. A. (2005). Policy-Making in the European Union. Fifth Edition, pp. 49-89. Oxford University Press

WALLACE, H.; WALLACE, W. Y POLLACK, M. A. (2005). Policy-Making in the European Union. Fifth Edition. Oxford University Press.

WEYLAND, K. (ed.) (2007). Bounded Rationality and Policy Diffusion: Social Sector Reform in Latin American. Princeton, NJ: Princeton University Press. 